\section{Nikotinpflaster, Vareniclin oder kombinierte Nikotinersatztherapie?}

\author{
Rauchen hat tiefgreifende gesundheitliche Auswirkungen. \\ Umso wichtiger ist die Suche nach Behandlungsformen, die \\ eine langzeitige Rauchabstinenz bewirken. T. B. Baker et al. \\ vom Center for Tobacco Research and Intervention in \\ Wisconsin/USA verglich 3 pharmakotherapeutische Ansätze \\ und fand keinen Unterschied hinsichtlich der Wirkung auf die \\ Rauchabstinenz.
}

JAMA 2016; 315: 371-379

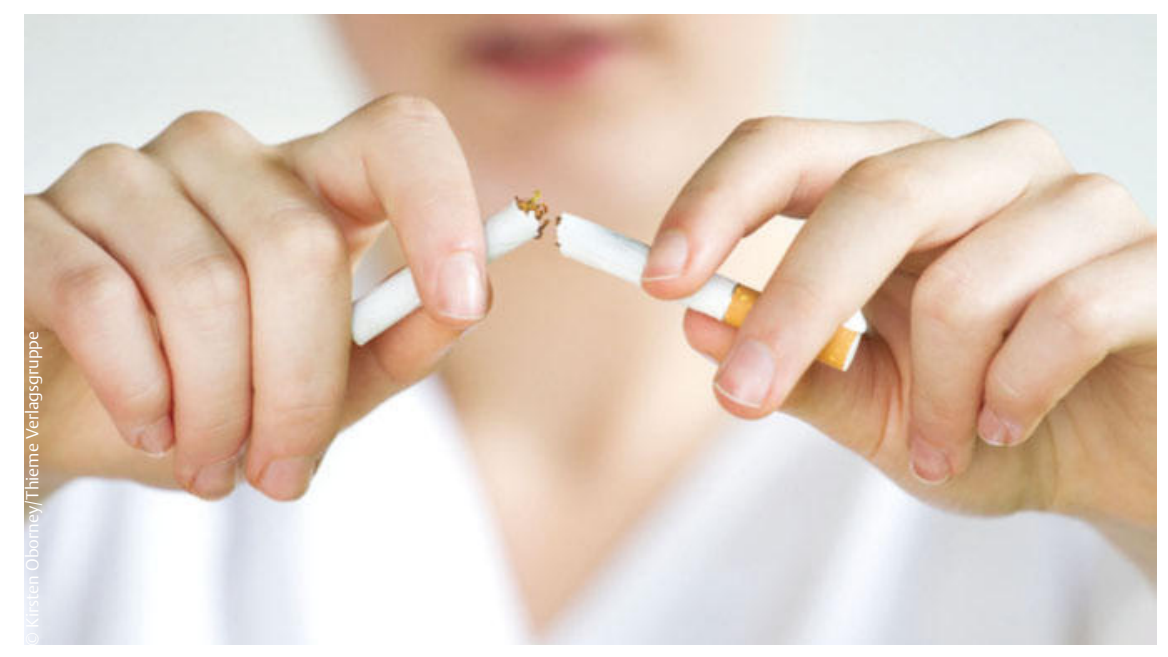

Das Cytisinderivat Vareniclin erleichtert über seine Wirkung auf Nikotinrezeptoren die Tabakentwöhnung. Es soll, ebenso wie die kombinierte Nikotinersatztherapie ( $\mathrm{k}$ NET), nach bisherigen Erkenntnissen der normalen Standardmedikation mit Nikotinpflastern in Monotherapie (NET) überlegen sein. Bisher liegt allerdings keine randomisierte klinische Studie vor, in der die Wirksamkeit von Vareniclin direkt mit der von k-NET verglichen wurde.

Die US-amerikanischen Forscher unternahmen daher eine Intent-to-Treat-Studie, in die sie 1068 Raucher aufnahmen, die mehr als 5 Zigaretten am Tag rauchten und mindestens 17 Jahre alt waren.Die Untersucher teilten sie randomisiert in 3 Gruppen für eine 12-wöchige Open-Label-Raucherentwöhnungstherapie ein:

1. Nikotinpflaster allein, je nach Rauchgewohnheit 7, 14 oder $21 \mathrm{mg}$ $(n=241)$,

2. $1 \mathrm{mg}$ Vareniclin 2-mal täglich allein (inklusive einer Prequit-Woche; $\mathrm{n}=424$ ) und
3. k-NET (Nikotinpflaster + täglich mind. 5 Nikotin-Lutschtabletten zu 2 oder $4 \mathrm{mg}$; $\mathrm{n}=421$ ).

Zusätzlich wurden 6 Beratungsgespräche angeboten.

Das primäre Zielkriterium bestand in der durch CO-Messung bestätigten 7-TageAbstinenz (Punktprävalenz, definiert als kein Zigarettenkonsum 7 Tage vor den Follow-up-Terminen nach eigener Aussage). Die sekundären Zielkriterien waren die nach eigener Aussage und durch CO-Messung bestätigte initiale Abstinenz und die anhaltende Abstinenz nach 26 Wochen sowie die Punktprävalenz-Abstinenz nach 4, 12 und 52 Wochen. Die Teilnehmer sollten Nebenwirkungen dem Forscherteam melden.

\section{Kein Unterschied bei Zielkriterien $\checkmark$}

566 (52\%) der Teilnehmer waren Frauen. Das mittlere Alter aller Teilnehmer lag bei $48,1 \pm 11,6$ Jahren. Im Durchschnitt rauchten sie seit 28,6 \pm 12 Jahren und konsumierten 17,0 \pm 8,3 Zigaretten am Tag. Von 917 (84\%) der Teilnehmer standen Follow-up-Daten über 12 Monate zur Verfügung.

Wie die statistische Auswertung mittels logistischer Regressionsanalyse ergab, unterschieden sich die Behandlungen weder nach 26 noch nach 52 Wochen hinsichtlich der Zielkriterien, einschließlich der Punkt-Prävalenz-Abstinenz nach 26 Wochen mit Abstinenzraten von 22,8\% unter Nikotinpflastern, 23,6\% unter Vareniclin und 26,8\% unter k-NET. Diese Raten unterschieden sich auch nach 52 Wochen nicht wesentlich (Nikotinpflaster 20,8\%; Vareniclin 19,1\%, kNET 20,2\%). Die Risikodifferenzen für Abstinenz betrugen nach 26 Wochen zwischen Pflaster und Vareniclin -0,76\%, zwischen Pflaster und k-NET -4,0\% und zwischen Vareniclin und k-NET -3,3\%.

Die Teilnehmer vertrugen alle Medikationen gut, allerdings verursachte Vareniclin häufiger Nebenwirkungen in Form von lebhaften Träumen, Schlaflosigkeit, Übelkeit, Verstopfung, Schläfrigkeit und Verdauungsstörungen als das Nikotinpflaster. Unter dem Nikotinpflaster allein und in Kombination traten hingegen häufiger Hautunverträglichkeiten auf.

\section{Fazit}

Die aufgrund der Erkenntnisse aus früheren Untersuchungen angenommene Überlegenheit von Vareniclin und der kombinierte Nikotinersatztherapie gegenüber dem Nikotinpflaster in Monotherapie findet in den Ergebnissen dieser Studie keine Bestätigung. Bei den zum Rauchverzicht motivierten erwachsenen Teilnehmern ergaben sich zwischen den 3 pharmakotherapeutischen Ansätzen keine deutlichen Unterschiede hinsichtlich der Quoten der Rauchabstinenz nach 26 oder 52 Wochen. Diese Ergebnisse werfen nach Ansicht der Autoren Fragen über die relative Wirksamkeit gegenwärtig eingesetzter Raucherentwöhnungstherapien auf. Sie weisen darauf hin, dass die Beratungsgespräche gut angenommen wurden und möglicherweise die Wirkung der Pharmakotherapie verwässert haben könnten, so die Autoren.

Dr. Volker Kriegeskorte, Buchloe 